\title{
Les Cestodes de quelques oiseaux de basse-cour dans la région de Cholon (Viet-Nam)
}

\author{
par Ch. JOYEUX et TRUONG-TAN-NGOG
}

\begin{abstract}
Au cours d'examens sanitaires effectués par l'un de nous (Truong-Tan-Ngoc) à Cholon, il a été examiné un grand nombre de volailles : poules, canards, pigeons, sacrifiés pour la consommation. Des cestodes ont été observés dans leurs intestins et le présent travail a pour but d'indiquer le pourcentage d'infestation ainsi que le degré de fréquence des diverses espèces.

A notre connaissance, un travail analogue n'a jamais été entrepris au Viet-Nam sur une aussi vaste échelle; on a simplement signalé des helminthes en se basant sur l'examen de quelques exemplaires, recueillis occasionnellement.

Comme on pourra s'en convaincre par les chiffres ci-dessous, il était matériellement impossible de récolter et de déterminer l'énorme quantité des cestodes observés : il a donc été prélevé une certaine quantité de ces vers, qui ont ensuite été identifiès. Des espèces rarement représentées ont $\mathrm{pu}$ ainsi nous échapper; mais notre but pratique était de connaitre les cestodes communément rencontrés dans la région.
\end{abstract}

\section{POULETS}

Environ 30.000 poulets ont été examinés. La proportion de cestodes était élevée, atteignant $90 \%$.

Il a èté prélevé environ 90 vers, répartis en 21 tubes, récoltés de mai à novembre 1949. Nous avons trouvé :

Raillietina (Raillietina) echinobothrida (Megnin, 1881).

Raillietina (Raillietina) tetragona (Molin, 1858). ............................

Cotugnia digonopora (Pasquale, 1890). $45,94 \%$

$29,73 \%$ $24,32 \%$

Les cestodes des poulets du Viet-Namı unt déjà fait l'objet de plusieurs publications. Dès 1897 , Gomy avait récolté Raillietina ( $R$. ecinobothrida) chez une poule de Saigon. Dans une collection portant sur les intestins de 25 poulets, recueillie par M. le Vétérinaire Bauche à Hué, en 1918-1919. remise à $M$. le Professeur Railliet d'Alfort, qui avait bien voulu nous en confier la détermination, nous avons identifié (1924):

Raillietina (Raillietina) echinobothrida

(Megnin, 1881)................... 13 fois

Raillietina (Raillietina) tetragona (Molin, 1858) ............................ II fois

Hymenolepis carioca (Magalhaes, 1908). 4 fois

Cotugnia digonopora (Pasquale, 1890).. 1 fois

Hymenolepis bauchei (Joyeux, 1924).... I fois

Cette dernière espèce était nouvelle.

En collaboration avec M. le Docteur-Vétérinaire E. Houdemer (1928), nous avons trouvé à Hanoï :

Raillietina (Raillietina) echinobothrida (Megnin, 1881).

Raillietina (Raillietina) tetragona (Molin, 1858).

\section{A Saigon :}

Amoebotaenia sphenoides (Railliet, 1892).

Hymenolepis cantaniana (Polonio, 1860).

On voit que les trois cestodes que nous signalons à Cholon étaient déjà connus au Viet-Nam.

$R$. (R.) echinobothrida et $R$. (R.) tetragona ont une vaste répartition géographique. Ils semblent exister dans tous les pays chauds, dans tout le bassin méditerranéen, mais disparaissent dans les climats tempérés. C'est ainsi qu'en France, très communs dans le Midi, ils se raréfient à mesure qu'on va vers le Nord. R. echinobothrida est le seul rencontré, et encore très rarement, dans le Centre. Ils sont absents de la région parisienne. Leur évolution est curieuse : les cysticercoïdes sont hébergés par des fourmis (Tetramorium semilaeve, André, pour $R$. echinobothrida dans la région de Marseille). Il est probable que c'est la fourmi adulte qui contracte le ver. Le poulet s'infeste en dévorant des fourmis qui hébergent le cysticercoïde. Dans le cas de $R$. tetragona, les larves ainsi absorbées commencent à se développer seulement dans la partie postérieure de l'intestin. Pour $R$. echinobothrida, la 
migration accomplie est plus compliquée. Les jeunes vers $s^{\prime}$ enfoncent dans la partie médiane de l'intestin, région du diverticule de $\mathrm{Meckel}$, et pénètrent profondément dans la paroi de cet organe en y occasionnant des lésions nécrotiques. Lorsque le cestode a atteint un certain dévëloppement, il sort de sa cavité, tombe dans la lumière intestinale et se trouve entraîné dans la partie postérieure de l'intestin, où il achève son développement.

Cotugnia digonopora existe en Europe orientale, en Asie, en Afrique tropicale. Son cycle évolutif est inconnu.

Ces cestodes peuvent avoir un rôle pathogène lorsqu'ils sont nombreux : diarrhée accompagnée de cachexie, parfois paralysie des pattes et accès épileptiformes.

\section{CANARDS}

Il a été examiné un nornbre de canards sensiblement égal à celui des poulets, soit à peu près 30.000 . La proportion de l'infestation par cestodes varie suivant l'âge des oiseaux; chezlle caneton, elle est de 0,1 à $0,5 \%$; chez le canard âgé, elle atteint 3 à $10 \%$.

Il a été prélevé environ 140 vers, répartis en 26 tubes, récoltés de mai à novembre 1949, à peu près aux mêmes dates que les cestodes des poulets. Il a été identifié :

Hymenolepis coronula (Dujardin, 1845)......................

Hymenolepis anatina (Krabbe, 1869)

Fimbriaria fasciolaris (Pallas, 1781)

Cotugnia, sp.................

Raillietina, sp $43,24 \%$ $30,03 \%$ $24,32 \%$ 1 exemplaire 1 exemplaire

Les exemplaires uniques de Cotugnia et de Raillietina étaient incomplets et indéterminables.

Les cestodes des canards domestiques d'Indochine semblent avoir été assez peu étudiés jusqu'à ce jour. Railliet, dans sa monographie (1924), cite seulement une ancienne observation de Neumann qui signale Hymenolepis (Drcpanidotacnia) lanceolata (Bloch, 1782), chez une oie domestique. Dans notre précédent travail, en collaboration avec E. Houdemer (1928), nous n'avons pas eu l'occasion d'identifier des cestodes de canards.

Les trois espèces dominantes : $H$. coronula, $H$. anatina et $r$. fasciolaris sont communément rencontrées chez le canard domestique. On les trouve aussi chez le canard sauvage ainsi que chez d'autres anatidés. Ils sont probablement répandus dans le monde entier. Ils ont pu être transportés en divers pays dans un but économique, ce qui est également le cas de nombreux helminthes d'animaux domestiques; en outre, les grandes migrations accomplies par leurs hôtes vivant en liberté ont, sans doute, contribué à leur dissémination. Cette vaste répartition géographique s'explique aussi par le fait que leurs larves cysticercoides sont euryxènes, c'est-à-dire évoluent chez de nombreuses espèces d'Entomostracés appartenant aux groupes Cyclops, Cypris, Candona, Diaptomus et formes voisines. Ainsi, le cycle évolutif peut $s$ accomplir à peu près dans tous les pays. Tous ces petits crustacés aquatiques s'infestent en ingérant les œufs contenus dans les déjections des anatidés. Ceux-ci contractent le cestode en absorbant les crustacés parasités. Nous avons mis en évidence un autre mécanisme (1929). Les entomostracés peuvent être consommés par des mollusques aquatiques, tels que des limnées (notamment Limnaea vulgaris Pfeiffer). S'ils hébergent des cysticercoïdes, ces derniers sont capables de demeurer longtemps vivants dans leur renflement stomacal et même de 's'y accumuler à miesure que de nouveaux crustacés sont ingérés par le mollusque. Celui-ci étant à son tour mangé par un canard, lui apporte tous les cysticercoïdes contenus dans son renflement stomacal, d'où infestation massive de l'oiseau. Nous avons pu observer ces faits en Normandie (ruisseau de Blonville-sur-Mer, Calvados) et à Marseille (pièce d'eau du parc Borély).

Les cestodes que nous venons de mentionner sont peu pathogènes; cependant lorsqu'ils sont en grand nombre, ils peuvent occasionner de la cachexie et même de l'obstruction intestinale.

\section{PIGEONS}

Il a été examiné 178 pigeons domestiques; la proportion d'infestation a atteint $85 \%$, chiffre élevé.

Il a été prélevé environ 70 vers, répartis en 10 tubes. Ces récoltes n'ont pu être faites aussi régulièrement que les précédentes. Quelques-unes sont du début de février et d'août, la majorité d'octobre et novembre 1949. Nous avons identifié :

\section{Raillietina (Raillietina) paucitesticulata}

(Fuhrmann, 1909)............. 42,5 \% Cotugnia taiwanensis (Yamaguti, 1935)... 28,56\% Raillietina (Raillietina) carpophagi (Joyeux

et Houdemer, 1927) ............ 14,28 \%

Hymenolepis serrata (Fuhr, 1906), v. birmanica (Megitt, 1924)........... 14,28\%

A notre connaissance, les cestodes des pigeons domestiques d'Indochine n'ont pas encore été étudiés. On a surtout décrit un certain nombre d'espèces se rapportant à des columbiformes sauvages. Il s'ensuit qu'ils sont assez mal connus et que certaines descriptions manquent de précision, ayant été faites sur un trop petit nombre d'exemplaires. 
Raillietina ( $R$.) paucitesticulata a été signalé depuis l'Indonésie jusqu'à Formose. Il existe aux Philippines une forme extrêmement voisine : Raillietina (Raillietina) sequens Tubangui et Masilungan (1937). Il est probable que ce groupe se trouve dans tout l'Extrême-Orient, présentant de légères variations morphologiques, suivant les pays et les hôtes. Cotugnia taiwanensis n'ost autre. que la forme " orientale de Cotugnia polyacantha Fuhrmann, 1909, connue dans le bassin méditerranéen et les régions voisines. Elle n'en diffère que par la taille des crochets; elle a été décrite au Japon. Nous avons récemment, en collaboration avec J.-G. Baer et J. Gaud, discuté, du point de vue zoologique, la morphologie et les affinités de $R$. paucitesticulata et de R. taiwanensis (1950).

Raillietina (R.) carpophagi a été décrite par nous chez Carpophaga oenea Salvad, dans la région de Thudaumont (Cochinchine). On voit qu'elle peut se trouver aussi chez le pigeon domestique. La morphologie de ces.deux cestodes cadre, à l'exception des dimensions de la poche du cirre: $0 \mathrm{~mm}$. $20 \mathrm{chez}$ le parasite du pigeon domestique, $0 \mathrm{~mm}$. $12 \mathrm{chez}$ celui de Carpophaga oenea. Cette légère différence es probablement à mettre sur le compte de la diversité des deux hôtes.

Hymenolepis serrata, var. birmanica a été décrite comme espèce type, chez des tourterelles, pigeons sauvages et domestiques dans l'Oural, le Turkestan, le Soudan anglo-égyptien, la Tunisie, l'Inde. Sa synonymie a été d'abord assez confuse. Meggitt (1924) en a signalé une variété chez le pigeon de Rangoon et l'a appelée birmanica. Les crochets sont un peu plus petits que dans le type. Nous avons retrouvé cette variété chez un pigeon de l'Inde, à Kasauli, mais sous forme d'échantillon incomplet. Plus tard, nous avons pu avoir des exemplaires en bon état, récoltés par $\mathrm{M}$. le Docteur-vétérinaire Houdemer, chez les tourterelles Streptopelia orjentalis (Lath.), Streptopelia chinensis tigrina (Temm.), Enopopelia tranquebarica humilis (Temm.), en Indochine. Nous avons ainsi établi, en collaboration avec J.-G. Baer (1935), l'histoire de cette espèce et de sa variété. On admet que la forme occidentale : H. serrata est l'espèce type, décrite la première; la variété birmanica se voit en Extrême-Orient.

Le cycle évolutif de tous ces cestodes de pigeons est inconnu. Nous pensons que les hôtes intermédiaires sont représentés par des invertébrés que peuvent consommer ces oiseaux, le cas échéant. Il y aurait probablement lieu de les rechercher dans les endroits fréquentés par les pigeons.

Leur rôle pathogène n'a pas été noté jusqu'à présent. Il est probablement analogue à celui que nous avons mentionné pour les cestodes de poulets et de canards, lorsque l'infestation est intense.

\section{TRAvauX cItés}

FUHRMANN (O.). - Neue Davaineiden. C. B. f. Ba t. u. Parasit 19, pp. $91121,1900$.

JOYEUX (Ch.). - Cestodes des poules d'Indochine. Ann. Parasit. II (4) pp. 314-318, 1924 .

JOYEUX (Ch.). - Procédé pour rechercher les cysticercoildes des petits crustacés. Ann. Parat. VI (2), pp, 112-115, 1929.

JOYELX (Ch.) et BAER (J. G.). - Cestodes' d'Indochine. Rev. zooi. suis. 42 (7), pp. 249-273, 1935.

JOYELX (Ch.), BAER (J. G. et GAUD (J.). - Recherches sur les cestodos d'Indochine ef sur quelques Diphyllobothrium (Bothriocéphales). Bull. Soc. Path. exot. XLIII, pp. 482-489, 1550.

JOYEUX (Ch.) et HOUDEMER (E.). - Recherches sur la faune helminthologique de l'Indochine (Cestodes et Trématodes). Ann. Parasitologie, V, pp. 289-309, 1928.

MEGGITT (F.) - - The tapeworms of the Rangoon Pigeon. Parasitology, V, pp. 303-312, 1924.

RRITITF.T (A.) - Les helminthes des animaux domestigues et de l'homme en Indochine. Bull. Soc. Zool, de France, XLIX, P. 588-608, 1924.

TUBANGI (M. A.) et MASILUNGAN (V. A.). - Tapeworm parasites of Phillppine Birds. Phil. Jour. of Sc. 62 (4), pp 409$436,1937$. 